2. Anwendung einer extremen Belastung an den gleichen Tieren. Diese Belastung sollte sowohl den Produktionsbedingungen entsprechen als auch die Grenze der Belastbarkeit der Tiere erreichen.

3. Nach Differenzierung der Tiere aufgrund der Überlebensrate in solche mit ausreichender und nicht ausreichender Adaptationsfähigkeit, muss anhand der Reaktion der gleichen Tiere unter mässiger Belastung festgestellt werden, ob eine hinreichend genaue Differenzierung in adaptationsfähige und nicht adaptatationsfähige Individuen aufgrund der ermittelten physiologischen Parameter möglich ist.

4. Durchführung einer standardisierten mässigen Belastung an einem grösseren Versuchstierbestand zur Ermittlung von Parametern als Basis für eine Selektion.

\title{
KONSTITUTIONSKRITERIEN BEI SCHWEINEN UNTERSCHIEDLICHER NUTZUNGSRICHTUNG UND GRÖSSE
}

J. UnShelm, H. Hohns, B. Oldigs und B. RühL. - Max-Planck-Institut für Tierzucht und Tievernährung, Arbeitsgruppe Konstitutionsforschung, Trenthorst über Bad Oldeslex (B.R. D.). - Institut für Tierzucht und Haustiergenetik der Universität Göttingen (B. R. D.). - Institut für Veterinärpathologie der Freien Universität Berlin West.

Bei Schweinen unterschiedlicher Nutzungsrichtung und Grösse (Deutsche Landrasse, Piétrain, Deutsches Weideschwein, Mangalica, Göttinger Miniaturschwein) wurden in Abhängigkeit vom Lebensalter vergleichende Untersuchungen über die Mast- und Schlachtleistung einschliesslich der Fleischbeschaffenheit sowie über die Morphologie und Physiologie wichtiger Organsysteme durchgeführt. Die Ergebnisse lassen erkennen, dass auch Schweine, die zu Rassen mit einem sehr geringen Muskelbildungsvermögen gehören, morphologische und physiologische Voraussetzungen aufweisen, die ungünstiger als die anderer Tierarten sind und die bisher zum Teil mit der einseitigen Selektion der Fleischschweine erklärt wurden. Andererseits konnte festgestellt werden, dass bei den Fleischschweinrassen und hier besonders bei den Piétrainschweinen hinsichtlich nahezu aller geprüften Organsysteme mit Ausnahme der Aktivität des Wachstumshormons Minderleistungen gegenüber den Fettsch weinrassen vorliegen oder zu erwarten sind. Aus den Ergebnissen wird gefolgert, dass die meist nicht sehr günstigen anatomischen und physiologischen Voraussetzungen beim Schwein durch die Selektion auf ein höheres Muskelbildungsvermögen noch weiter verschlechtert wurden. Es wird vorgeschlagen, mehrere physiologische Parameter zunächts versuchsweise und dann routinemässig im Rahmen der Mastleistungsprüfungen zu erfassen. Gleichzeitig könnte dabei der Erblichkeitsantiel von Konstitutionsmerkmalen geschätzt werden. Das wäre eine wichtige Voraussetzung für eine gezielte züchterische Beeinflussung der Konstitution und damit der Widerstandsfähigkeit des Schweines.

\section{RELATIONS ENTRE ILES RÉACTIONS DU PORC A UN CHOC THERMIQUE ET LA QUALITÉ DE LA VIANDE}

J. Charpentier, G. Monin et L. Ollivier*. - Station de Recherches sur la viande, 63 - Theix. -_. * Station de Génétique quantitative et appliquée, I. N. R. A., 78-Jouy-en-Josas (France).

269 porcs Large White (LW $\times$ LW) (échantillon A) et 296 porcs (échantillon B) appartenant aux races ou croisements suivants, Piétrain $(\mathrm{P} \times \mathrm{P})$, Piétrain $\times$ Large White $(\mathrm{P} \times \mathrm{LW})$, Porc Blanc de l'Ouest $\times$ Piétrain $(\mathrm{PBO} \times \mathrm{P})$ et Porc Blanc de l'Ouest $\times$ Large White $(\mathrm{PBO} \times \mathrm{LW})$, ont été soumis à une température de $4^{\circ} \mathrm{C}$ pendant vingt minutes, une semaine avant leur abattage, à environ Ioo $\mathrm{kg}$ de poids vif. La température musculaire, le rythme respiratoire et le rythme cardiaque ont été enregistrés au début et à la fin du traitement thermique. Des mesures de couleur, de rétention d'eau et de $\mathrm{pH}$ ont été prises sur le jambon, 24 heures après l'abattage. La distance entre l'élevage et l'abattoir était de $30 \mathrm{~km}$ pour A et $90 \mathrm{~km}$ pour B. Les animaux disposaient de I 2 à I 8 heures de repos entre leur arrivée à l'abattoir et l'abattage. L'expérience s'est déroulée entre août et novembre I969 pour A et mai et décembre I970 pour B. 